\title{
The Effects of TMP Treatment and High Fat Diet on Bone Fracture Healing
}

Samuel Zike ${ }^{1}$, Jeffery Nielsen ${ }^{3,4,5}$, Caio de Andrade Staut ${ }^{1}$, Vincent Alentado ${ }^{2}$, Ushashi Dadwal $^{1}$, Christopher Dalloul ${ }^{1}$, Abduallah Elsayed ${ }^{1}$, Nicholas Hux ${ }^{1}$, Nikhil Tewari ${ }^{1}$, Mustafah Shaikh $^{1}$, Sarah Myers ${ }^{1}$, Murad Nazzal ${ }^{1}$, Hanisha Battina ${ }^{1}$, Rachel Blosser ${ }^{1}$, Jiliang Li ${ }^{6}$, Stewart Low $^{3,5}$, Mary Niedrauer ${ }^{3}$, Philip Low ${ }^{4,5}$, Melissa Kacena ${ }^{1,7}$

Departments of ${ }^{1}$ Orthopaedic Surgery and ${ }^{2}$ Neurological Surgery, Indiana University School of Medicine, Indianapolis, IN; ${ }^{3}$ Novosteo Inc., West Lafayette, IN; Departments of ${ }^{4}$ Chemistry and ${ }^{5}$ Medicinal Chemistry and Pharmacology, Purdue University, West Lafayette, IN; Department of ${ }^{6}$ Biology, Indiana University Purdue University, Indianapolis, Indianapolis, IN; ${ }^{7}$ Richard L. Roudebush VA Medical Center, Indianapolis, IN

Delayed and impaired bone fracture healing are associated with diabetic populations. This is a challenging problem for orthopaedic surgeons especially in the US where the percentage of type 2 diabetic patients continues to climb at an alarming rate. Limited treatment options exist for orthopaedic surgeons to improve fracture healing, and the most commonly used therapies involve placement of proteins (bone morphogenetic protein), graft tissue, or demineralized bone matrix at the fracture site. We have previously demonstrated that local administration of the main megakaryocyte growth factor, thrombopoietin, enhances bone healing. Here we demonstrate the utility of systemically administering thrombopoietin mimetic peptides (TMPs) to improve impaired fracture healing in a mouse model of type 2 diabetes. Briefly, 120 male mice on a C57BL/6 background were placed on a low fat diet (LFD) or high fat diet (HFD) for 12 weeks prior to undergoing a surgically created femoral fracture. Mice were treated with 33 $\mathrm{nmol} / \mathrm{kg}$ of TMP or saline immediately after surgery and daily for the following week. Mice were euthanized at 1, 2, and 4 weeks post-surgery ( $n=10 /$ group). Here, we confirmed that HFD resulted in impaired fracture healing. We also showed accelerated bone union and increased callus formation in TMP treated mice compared to saline groups, irrespective of diet $(p<0.05)$. Among TMP groups that were fed either a HFD or LFD, the HFD TMP group showed greater improvements in bone healing compared to the HFD saline control mice. Further study on TMP should include alternative routes of administration and providing treatment when a surgical repair appears to be deteriorating. Although there is more to be understood about the clinical importance and mechanism by which systemic TMP treatment enhances fracture healing, these data appear promising. 